Reviving the Philosophy of Geometry David Corfield

Department of Philosophy, University of Kent 


\section{Reviving the Philosophy of Geometry}

\section{Introduction}

Leafing through Robert Torretti's book Philosophy of Geometry from Riemann to Poincaré (1978), it is natural to wonder why, at least in the Anglophone community, we have little activity meriting this name today. Broadly speaking, we can say that any philosophical interest in geometry shown here is directed at the appearance of geometric constructions in physics, without any thought being given to the conceptual development of the subject within mathematics itself. This is in part a result of a conception we owe to the Vienna Circle and their Berlin colleagues that one should sharply distinguish between mathematical geometry and physical geometry. Inspired by Einstein's relativity theory, this account, due to Schlick and Reichenbach, takes mathematical geometry to be the study of the logical consequences of certain Hilbertian axiomatisations. For its application in physics, in addition to a mathematical geometric theory, one needs laws of physics and then 'coordinating principles' which relate these laws to empirical observations. From this viewpoint, the mathematics itself fades from view as a more or less convenient choice of language in which to express a physical theory. No interest is taken in which axiomatic theories deserve the epithet 'geometric.'

However, in the 1920s this view of geometry did not go unchallenged as Hermann Weyl, similarly inspired by relativity theory, was led to very different conclusions. His attempted unification of electromagnetism with relativity theory of 1918 was the product of a coherent geometric, physical and philosophical vision, inspired by his knowledge of the works of Fichte and Husserl. While this unification was not directly successful, it gave rise to modern gauge field theory. Weyl, of course, also went on to make a considerable contribution to quantum theory. And while Einstein gave initial support to Moritz Schlick's account of his theory, he later became an advocate of the idea that mathematics provides important conceptual frameworks in which to do physics:

Experience can of course guide us in our choice of serviceable mathematical concepts; it cannot possibly be the source from which they are derived; experience of course remains the sole criterion of the serviceability of a mathematical construction for physics, but the truly creative principle resides in mathematics. (Einstein 1934, p. 167)

We may imagine then that an important chapter in any sequel to Torretti's book would describe both Reichenbach's and Weyl's views on geometry. This is done in Thomas Ryckman's excellent The Reign of Relativity (2005), where the author also discusses further overlooked German-language philosophical writings on geometry from the 1920s, this time by Ernst Cassirer. Cassirer's extraordinary ability to assimilate the findings of a wide range of disciplines sees 
him discuss the work of important mathematicians such as Felix Klein, Steiner, Dedekind and Hilbert.

Ryckman ends his book with a call for philosophical inquiry into what sense a "geometrized physics" can have, to emulate the above mentioned work from the interwar period. And he is not alone in thinking that this was a golden age. There is now an impressive concentration on this era. Today, in Ryckman and similar-minded thinkers, such as Michael Friedman and Alan Richardson (see the contributions of all three in Domski and Dixon (2010)), we find fascinating discussions about these themes. However these discussions by themselves are unlikely to give rise today to the kind of primary work that they are studying from the past. It is one thing to make a careful, detailed study of the interweaving of philosophy, mathematics and physics of a period, quite another to begin to take the steps necessary for a revival of such activity.

Michael Friedman (2001) points out two connected, yet somewhat distinct, activities dealing with mathematical physics which might be called 'philosophical'. One termed 'metascientific' is much as Weyl does, reconceiving the idea of space and thereby generating foundational advances. Metascience is typically done by philosophically informed scientists, such as Riemann, Helmholtz, Poincaré, and Einstein. By contrast, the other activity is much as Cassirer and the Vienna Circle did, reflecting on the broader questions of the place of mathematics and science in our body of knowledge in light of important events in the histories of those practices. While there spontaneously arises work of the first kind in any era, work of the second kind requires a philosophical orientation which may be lost. One very obvious difference is that today we have so few philosophers emulating Cassirer by keeping abreast of the mathematics of the recent past. This simply must change if we are to generate the forms of discussion to parallel those of the 1920s. For too long, philosophy has thought to constrain its interest in any current mathematical research largely to set theory, when it has long been evident that it offers little or nothing as far as many core areas of mathematics are concerned, and especially the mathematics needed for physics. Casting the differential cohomology of modern quantum gauge theory in set theoretic clothing would do no favours to anyone. So, with some notable exceptions, such as Marquis (2008) and McLarty (2008), we let the bulk of mainstream mathematical research pass us by.

However there are reasons to be hopeful. I shall argue in this chapter that our best hope in reviving a 1920s-style philosophy of geometry lies in following what has been happening at the cutting edge of mathematical geometry over the past few decades, and that while this may appear a daunting prospect, we do now have ready to hand a means to catch up rapidly. These means are provided by what is known as cohesive homotopy type theory.

Univalent foundations, or plain homotopy type theory (cf. Awodey's and Shulman's chapters), provide the syntax for theories which can be interpreted within $(\infty, 1)$-toposes, a generalisation of the ordinary notion of toposes. The basic shapes of mathematics are now taken to be the so-called 'homotopy $n$ types'. However these are not sufficient to do what needs to be done in modern geometry, and especially in the geometry necessary for modern physics, since 
we need to add further structures to express continuity, smoothness, and so on. As we add extra properties and structures to $(\infty, 1)$-toposes, characterised by qualifiers - local, $\infty$-connected, cohesive, differentially cohesive - increasing amounts of mathematical structure are made possible internally. The work of Urs Schreiber (2013) has shown that cohesive $(\infty, 1)$-toposes provide an excellent environment to approach Hilbert's sixth problem on axiomatising physics, allowing the formulation of relativity theory and all quantum gauge theories, including the higher-dimensional ones occurring in string theory.

Cohesiveness in this sense arose from earlier formulations of the notion in the case of ordinary toposes by William Lawvere (2007), motivated in turn by philosophical reflection on geometry and physics. Schreiber's claim, however, is that for these concepts to take on their full power they must be extended to the context of higher topos theory, that is the theory of $(\infty, 1)$-toposes, where differential cohomology finds its natural setting. Now, rather than the mathematics necessary for physics being viewed, as it often is at present from a set theoretic foundation, as elaborate and unprincipled, we can see the simplicity of the necessary constructions through the universal constructions of higher category theory.

In a single article it will only be possible to outline the kind of work that is necessary to fill in the spaces we have left ourselves. There is important interpretative work to do already in making sense of plain homotopy type theory, so here I can only indicate further work to be done. At the same time, in that mathematics finds itself once again undergoing enormous transformations in its basic self-understanding, it is important as philosophers to take this opportunity to remind ourselves that we should provide an account of mathematical enquiry where such changes are to be expected. It is striking that Hegel should be found informing both those wishing to characterise the dynamic growth of mathematics, and those striving to refashion the very concepts of modern geometry itself.

\section{Current Geometry}

If any reassurance is needed that geometry is alive and well today, one need only look at the variety of branches of mathematics bearing that name which are actively being explored:

Algebraic, differential, metric, symplectic, contact, parabolic, convex, Diophantine, tropical, conformal, Riemannian, Kähler, Arakelov, analytic, rigid analytic, global analytic, ...

Continuing our search, we find noncommutative versions of some of the above, 'derived' versions, and so on. Indeed, there has never been so much 'geometric' research being carried out as there is today, from constructions that Gauss or Riemann might have recognised to ones which would seem quite foreign. So the question arises of whether this list provides just a motley of topics which 
happen to bear the same name, or whether there is something substantial that is common to all of them, or at least many of them.

Evidence for the latter option comes from people still making unqualified use of 'geometry' and its cognates to mean something. Some such uses are informal, as in the following example:

The fundamental aims of geometric representation theory are to uncover the deeper geometric and categorical structures underlying the familiar objects of representation theory and harmonic analysis, and to apply the resulting insights to the resolution of classical problems. (MRSI 2014)

Other uses are technical, such as where Jacob Lurie (2009) uses the term 'geometry' to name a certain kind of mathematical entity, here a small $(\infty, 1)$-category with certain additional data. The question then arises as to what features of these structures make Lurie single them out as geometries. At first glance this seems a rather technical matter. Let us return to it once we have some motivation from the past.

Something that would have seemed novel to Gauss and Riemann, and which might give rise to doubts concerning the unity of geometry, is the thorough injection of spatial ideas brought into algebraic geometry by Alexandre Grothendieck in the 1960s. By the late 1800s it was already known of the collection of complex polynomials, $\mathbb{C}[z]$, that the space these functions are defined on could be recaptured from the algebraic structure of the collection itself. $\mathbb{C}[z]$ forms a ring, and it is possible to construct an associated space from points corresponding to its maximal ideals. These are the ideals generated by $(z-a)$, for each $a \in \mathbb{C}$. Picking up on the complex function field/algebraic number field analogy of Dedekind and Weber, as developed by Weil in his Rosetta Stone account (see Corfield 2003, Ch. 4), it was then shown that even apparently non-functional rings, such as the ring of integers and others encountered in arithmetic, might be treated likewise. Grothendieck's scheme theory (see McLarty 2008) provided such a space, in the case of the integers denoted $\operatorname{Spec}(\mathbb{Z})$, again constructed out of prime ideals. Now an integer is considered as a function defined at each prime, a point in $\operatorname{Spec}(\mathbb{Z})$, as the function $n(p) \equiv n(\bmod p)$. Where this differs from the complex function case is that while the values of 'integer-as-function' still land in a field, here the field varies according to the point where the function is evaluated, $\mathbb{F}_{p}$ as $p$ varies. This suggests a space whose points are not identical.

This attempt to geometrise arithmetic is not an empty game. It feeds through to mathematical practice as can readily be discovered thanks to the growth of online informal discussion.

I like to picture $\operatorname{Spec} \mathbb{Q}$ as something like a 2 -manifold which has had all its points deleted. The extra complication is that what we think of as the points are actually very small circles. So it's really a three manifold with all of the loops inside it deleted. 
For example, let's look first at function fields. Spec $\mathbb{C}[z]$ is just the complex line $\mathbb{C}$. As we start inverting elements of $\operatorname{Spec} \mathbb{C}[z]$, as we must do to make $\operatorname{Spec} \mathbb{C}(z)$, the effect on the spectrum is to remove bigger and bigger finite sets of points. The limit is where we remove all the points and we're just left with some kind of mesh.

If we had started with a Riemann surface of genus $g$, then we'd be left with a mesh of genus $g$, a surface sewn out of the cloth from which fly screens for windows are made. If we want to recover the original surface from the surface mesh, we just put it out back in the shed for a while and let the mesh fill up with dirt. This is just the familiar fact that a (smooth compact, say) Riemann surface can be recovered from the field of meromorphic functions on it.

If we replace $\mathbb{C}$ by a finite field $\mathbb{F}$, then everything is the same but what we thought of as the point is now a very small circle, and so our original surface reveals itself to be a 3-manifold fibered over a very small circle when we zoom in. And when we delete points, we're really deleting not just single-valued sections of this fibration but also multivalued sections. So Spec $\mathbb{F}[z]$ is some kind of 3 -manifold fibered over the circle with all the loops over the base circle deleted.

For the passage from $\mathbb{Z}$ to $\mathbb{Q}$, I don't have anything better to say than that it's sort of the same but there's no base circle. We're just removing lots of loops from a 3-manifold. Maybe some should be seen as bigger than others, corresponding to the fact that there are prime numbers of different magnitudes. (Borger 2009)

Here we see Borger passing across each of the three columns of Weil's Rosetta Stone.

Now, not only do we find a geometrised arithmetic, but these ideas and constructions are the structural cousins of those appearing in cutting-edge physics, as we see in this comment by David Ben-Zvi:

...the geometric analog of a number field or function field in finite characteristic should not be a Riemann surface, but roughly a surface bundle over the circle. This explains the "categorification" (need for a function-sheaf dictionary, which is the weak part of the analogy) that takes place in passing from classical to geometric Langlands - if you study the corresponding QFT on such three-manifolds, you get structures much closer to those of the classical Langlands correspondence. (Ben-Zvi 2014)

So we have both widespread current interest in classically geometric areas of mathematics and geometric approaches to other areas, including arithmetic, and ways of thinking about the subject matter expressed, at least informally, in a very visual language. Geometry as a whole is something larger that that which has application in mathematical physics, and applied mathematics more 
generally. Similar structures are now found to lie at the heart of number theory. But how to go about saying something satisfactorily general about geometry?

One might throw up one's hands at the task of bringing this wealth of subject matter under the umbrella of a straightforward description. To the extent that people try to do this it is largely left to the doyens of mathematics. For example, Sir Michael Atiyah writes

Broadly speaking, I want to suggest that geometry is that part of mathematics in which visual thought is dominant whereas algebra is that part in which sequential thought is dominant. (Atiyah 2003, p. 29)

Such a distinction is reminiscent of Kant, for whom space and time were considered to be forms of sensibility, and yet Atiyah continues

This dichotomy is perhaps better conveyed by the words "insight" versus "rigour" and both play an essential role in real mathematical problems. (Atiyah 2003, p. 29)

A more careful treatment is required here, since there seems nothing to object to in the idea of 'rigorous geometry' or 'algebraic insight'. We need to turn back the clock to when philosophical research directed itself towards then current geometry.

\section{Regaining the Philosophy of Geometry}

What led to the demise of the philosophy of geometry in the English-speaking world? I think this can be attributed largely to the success of logical empiricism. Many of those dispersed from Germany and Austria in the 1930s were accepted into the universities of the USA, welcomed by existing empiricists such as Ernst Nagel. In a long paper published in 1939, Nagel uses the history of projective geometry to explain the new understanding of then modern axiomatic mathematics:

It is a fair if somewhat crude summary of the history of geometry since 1800 to say that it has led from the view that geometry is the apodeictic science of space to the conception that geometry, in so far as it is part of natural science, is a system of "conventions" or "definitions" for ordering and measuring bodies. (Nagel 1939, p. 143)

The distinction between a pure and an applied mathematics and logic has become essential for any adequate understanding of the procedures and conclusions of the natural sciences. (Nagel 1939, p. 217) 
So axiom systems are proposals for stipulations. As pure mathematics they are to be studied for their logical properties. Some of them may be found to be well adapted to allow the expression of scientific laws, which may then be used in applied sciences. This is made possible by coordinating principles which tie the scientific laws to empirical measurements. For example, Riemannian geometry allows for the expression of Einstein's field equations, which can be coordinated to observation by stipulating that light follows null geodesics.

While now the ideational content of mathematics is left to one side, other tasks fall to the philosopher of mathematics:

...the concepts of structure, isomorphism, and invariance, which have been fashioned out of the materials to which the principle of duality is relevant, dominate research in mathematics, logic, and the sciences of nature. (Nagel 1939, p. 217).

Had philosophers at least heeded this, more attention might have been paid to category theory, the language par excellence of structure, isomorphism and invariance, which emerged shortly after Nagel's paper (Corfield 2015). As it was, a uniform treatment of mathematics as the logical consequences of definitions, or of the set theoretic axioms came to prevail.

In the process, as Heis (2011) argues, two lines of thought from earlier in the century were being ruled out, each responding to other nineteenth century developments in geometry:

1. What could be saved of Kantian philosophy given the appearance of nonEuclidean geometry, and then Riemannian geometry? What are the conditions for spatial experience?

2. How should we understand the ever changing field of geometry given the introduction of ideal elements, imaginary points, and so on?

Let us take each of these in turn.

\subsection{Weyl: The Essence of Space}

With an ever-expanding variety of geometries emerging through the nineteenth century, it became implausible to maintain with Kant that our knowledge of Euclidean geometry is a priori. Helmholtz had argued that because empirical measurement requires that objects undergo only 'rigid motions', we can work out which geometries are presupposed by our physics. He concluded that only those spaces which possessed the property of constant curvature were permissible. With the contribution of the technical expertise of Sophus Lie, this line of research resulted in the Helmholtz-Lie theorem, characterising Euclidean, elliptic and hyperbolic geometries.

Research such as this was certainly discussed by philosophers. Indeed, Russell, and later Schlick and Reichenbach responded to Helmholtz's work, but perhaps the most profound response came from Hermann Weyl. After the success of Einstein's general theory of relativity, Helmholtz's results were evidently 
far too limited. Weyl, inspired by Husserl and perhaps more profoundly by Fichte (see Scholz 2005), sought to discern "the essence of space." In a letter to Husserl, he wrote

Recently, I have occupied myself with grasping the essence of space [das Wesen des Raumes] upon the ultimate grounds susceptible to mathematical analysis. The problem accordingly concerns a similar group theoretical investigation, as carried out by Helmholtz in his time ... (Ryckman 2005, p. 113)

Weyl conceived of spaces in which it was only possible to compare the lengths of two rods if they were situated at the same point.

Only the spatio-temporally coinciding and the immediate spatialtemporal neighborhood have a directly clear meaning exhibited in intuition. (Weyl 'Geometrie und Physik', quoted in (Ryckman 2005, p. 148)

Along the lines of Helmholtz and Lie, this led him to prove a group theoretic result: The only groups satisfying certain desiderata (involving the "widest conceivable range of possible congruence transfers" and a demand for a single affine connection), are the special orthogonal groups of any signature with similarities, $G \simeq S O(p, q) \times \mathbb{R}^{+}($Scholz 2011).

There is an interesting story to be told here of how Einstein and other physicists found implausible the possibility allowed by this geometry that rods of identical lengths as measured at one point, if transported along different paths to a distant point might have different resulting lengths. One usually tells the story of how the beauty of the mathematics got the better of Weyl, and how physicists eventually uncovered what was good about the idea whilst modifying his original idea to allow a $U(1)$ gauge group. This story needs to be told in a much more nuanced way (see Giovanelli 2013), and in any case is complicated by the survival of Weyl's original idea in forms of conformal gauge theory.

In any case, Weyl himself later became sceptical of this kind of mathematical speculation about the geometry required for physics that had so consumed him in his earlier years. With the demise of other philosophical attempts to study our a priori geometric intuition, for example, Carnap's doctoral thesis on how our intuitive concept of space was required to be $n$-dimensional topological space, such attempts largely come to an end. We will take a look at more recent 'meta-scientific' kinds of work, but first let us turn to Friedman's other form of philosophical research.

\subsection{Cassirer: Beyond intuition}

In an unusual paper, published the year before his death, Ernst Cassirer (1944) argued for an important connection to be seen between Felix Klein's Erlanger program and our everyday perception. Where Klein had given a presentation of many forms of geometry as the study of invariants of space under the action 
of groups of symmetry, Cassirer saw the seeds for this idea in our abilities to perceive the invariant size, colour and shape of objects under varying viewing conditions. Now evidently these abilities are rooted in our distant evolutionary past, and yet the full-blown mathematical idea had only crystallised in modern mathematical thinking around 1870.

Klein's ideas on geometry marked an important stage in the course of a revolutionary century for geometric thought. Not only had the range of geometries been extended from the single Euclidean geometry to hyperbolic and elliptical forms, but there had been many kinds of extension of the notion of space by the introduction of elements that seemed to lead us away from the intuitively familiar. For example, the non-degenerate conic sections had been unified as curves of degree 2 in complex projective space, brought about by the addition of 'points at infinity' and complex coordinates. Now all circles were seen to pass through two imaginary points at infinity, and so 'intersect' there.

Such forays beyond the intuitive led those wishing to retain what they took to be valuable in Kant to take a different tack. As Heis (2011) convincingly shows, the neo-Kantian Cassirer had to come to terms with just such developments. This is evident in his later work:

It is hence obvious that mathematical theories have developed in spite of the limits within which a certain psychological theory of the concept tried to confine them. Mathematical theory ascended higher and higher in order to look farther and farther. Again and again it ventured the Icarian flight which carried it into the realm of mere "abstraction" beyond whatever may be given and represented in intuition. (Cassirer 1944, p. 24)

But then without any firm rootedness in intuition, what provides us with guidance that our "Icarian flights" are heading in the best direction? This problematic runs through Cassirer's career, and is answered by the unity of the history of the discipline.

Though a properly Neo-Kantian philosophy of mathematics will appreciate that mathematics itself has undergone fundamental conceptual changes throughout its history, such a philosophy will also have to substantiate the claim that the various stages in the historical development of mathematics constitute one history...we can say that they [mathematicians] were studying the same objects only because we can say that they are parts of the same history. (Heis 2011, p. 768)

It is worth quoting Cassirer at length on this point:

...it is not enough that the new elements should prove equally justfied with the old, in the sense that the two can enter into a connection that is free from contradiction - it is not enough that the new should take their place beside the old and assert themselves in 
juxtaposition. This merely formal combinability would not in itself provide a guarantee for a true inner conjunction, for a homogeneous logical structure of mathematics. Such a structure is secured only if we show that the new elements are not simply adjoined to the old ones as elements of a different kind and origin, but the new are $a$ systematically necessary unfolding of the old. And this requires that we demonstrate a primary logical kinship between the two. Then the new elements will bring nothing to the old, other than what was implicit in their original meaning. If this is so, we may expect that the new elements, instead of fundamentally changing this meaning and replacing it, will first bring it to its full development and clarification. (Cassirer 1957, p. 392)

If one can hear an overtone of Hegelian thought here, this is not surprising. In the introduction to this third volume of The Philosophy of Symbolic Forms Cassirer explained the debt to Hegel as shown by the subtitle of the book - The Phenomenology of Knowledge:

The truth is the whole - yet this whole cannot be presented all at once but must be unfolded progressively by thought in its own autonomous movement and rhythm. It is this unfolding which constitutes the being and essence of science. The element of thought, in which science is and lives, is consequently fulfilled and made intelligible only through the movement of its becoming. (Cassirer 1957, p. xiv)

This line of thought sits very happily with the idea that important developments in a discipline allow its history to be written in such a way that it makes best sense of what was only obscurely seen in the past or of what became the means to overcome perceived obstacles or limitations, in other words, a history of rational unfolding out of an older stage. As I argue in Corfield (2012), we find this position very well expressed by the moral philosopher Alasdair MacIntyre. A similar idea is expressed by Friedman's 'retrospective' rationality (2001).

In the Anglophone revival (Friedman, Ryckman, Richardson, Heis, and Everett) of interest in Cassirer of recent years there has been particular focus on the place of the 'constitutive' and the 'regulative' in his account of the progress of science. This amounts to rival interpretations of the relative importance for Cassirer of the prospective overcoming of limitations within a discipline and the retrospective rationalisation of its course, eventually as seen from an ideal future point. However these debates turn out, it is intriguing then to see what we might call a further strand added in the 1944 paper, that in mathematics we may devise concepts which owe their origin to unnoticed cognitive structures. In the case of the Erlanger Program

...the mathematical concepts are only the full actualisation of an achievement that, in a rudimentary form, appears also in perception. Perception too involves a certain invariance and depends upon it for its inner constitution. (Cassirer 1944, p. 17) 
Taken together, we can see in the work of Weyl and Cassirer just how far we are here in attitude towards mathematical geometry from what was bequeathed to us by the logical empiricists. Heis quotes Hans Reichenbach:

It has become customary to reduce a controversy about the logical status of mathematics to a controversy about the logical status of the axioms. Nowadays one can hardly speak of a controversy any longer. The problem of the axioms of mathematics was solved by the discovery that they are definitions, that is, arbitrary stipulations which are neither true nor false, and that only the logical properties of a system - its consistency, independence, uniqueness, and completeness - can be subjects of critical investigation. (Heis 2011, p. 790)

He very aptly writes "One could hardly find a point of view further from Cassirer's own" (Heis 2011, p. 790). Indeed so, but now to be true to Cassirer's spirit we should try to work out our own position on the rationality of mathematical enquiry in the process of coming to frame what has been happening in the mathematics of the recent past. That this has typically not been felt to be a requirement of philosophy makes this no easy task, but we should try to make a start anyway.

\section{Capturing Modern Geometry}

Even to summarise one particular line of development here will not be easy. Along with Grothendieck's invention of scheme theory, mentioned in section 2, we would also need to talk about topos theory. This could take the form of a story of natural unfolding. Indeed, we could report the originator's own words.

...one can say that the notion of a topos arose naturally from the perspective of sheaves in topology, and constitutes a substantial broadening of the notion of a topological space, encompassing many concepts that were once not seen as part of topological intuition... As the term "topos" itself is specifically intended to suggest, it seems reasonable and legitimate to the authors of this seminar to consider the aim of topology to be the study of topoi (not only topological spaces). (Grothendieck and Verdier 1972, p. 302)

However, for the purposes of this chapter, we need to race forward to much more recent work. Jacob Lurie motivates his 'Structured Spaces' paper (2009) by means of an account of the passage to less restricted forms of Bézout's theorem. This is a result that goes back to the eighteenth century, involving just the kind of achievement of unity through addition of ideal elements that interested Cassirer. While it was known to Newton that the number of real solutions to the intersection of a pair of plane curves was bounded by the product of their degrees, by the nineteenth century we find a form of the result that states 
that two complex projective plane curves of respective degrees $m$ and $n$ which share no common component have $m \cdot n$ points of intersection, counted with multiplicity. Any two non-identical conics meet four times, including at those imaginary points at infinity in the case of two circles that were mentioned in the previous section.

Lurie takes this result up, looking to understand it in terms of cohomology and the cup product of fundamental classes of the curves, which corresponds to the class of their intersection. Since this method does not work for nontransverse intersections, using Grothendieck's constructions we then turn to 'nonreduced' schemes. Further, according to Lurie, we should look at an Euler characteristic involving the dimension of the local ring of the scheme-theoretic intersection plus various corrections.

Now an interesting thing happens when we attempt to retain the fundamental result $[C] \cup\left[C^{\prime}\right]=\left[C \cap C^{\prime}\right]$ in the very general setting where there may even be coinciding components. Here we need derived algebraic geometry.

To obtain the theory we are looking for, we need a notion of generalized ring which remembers not only whether or not $x$ is equal to 0 , but how many different ways $x$ is equal to 0 . One way to obtain such a formalism is by categorifying the notion of a commutative ring. That is, in place of ordinary commutative rings, we consider categories equipped with addition and multiplication operations (which are encoded by functors, rather than ordinary functions). (Lurie 2009, p. 3)

Lurie is drawing attention here to the passage from the proposition 'Is $x$ equal to 0 ?' to the set of ways in which it is equal. To do so is to take the first step up an infinitely tall ladder of weakenings of identity. In (Corfield 2003, Ch. 10) I give an account of categorification, the replacement of set by category by 2category. In the dozen or so years since my book, the emphasis has swung round to the groupoid version of this ladder, where sets become groupoids become 2groupoids, etc. Lurie, in particular, was instrumental in this change in showing that most constructions of ordinary category theory have their analogues in the $(\infty, 1)$ setting, where instead of hom-sets between objects, we have $\infty$-groupoids.

Now, since when dealing with a pair of coinciding lines, we need to make identifications in the form of isomorphisms, we find that

These isomorphisms are (in general) distinct from one another, so that the categorical ring $C$ "knows" how many times $x$ and $y$ have been identified. (Lurie 2009, p. 4)

Of course, we never stop with a single step up this ladder, and eventually we seek further generalized forms of ring, such as $E_{\infty}$-ring spectra and simplicial commutative rings. The key lesson here is that to retain a simple formulation, we have to change our framework, for one thing here to allow homotopic weakening. In Cassirerian terms, this is forced upon us by the natural unfolding of the discipline. And it is not just algebraic geometry that demands this richer notion 
of space, so does physics. The moduli spaces of today's gauge field theories are often stacks, such as the moduli stack of flat connections for some gauge group. Higher gauge theory requires similar homotopic weakening to higher stacks (Schreiber 2013).

Naturally, Lurie is not a lone voice in calling for this change of outlook. Betrand Toën likewise gives an account of derived algebraic geometry

Derived algebraic geometry is an extension of algebraic geometry whose main purpose is to propose a setting to treat geometrically special situations (typically bad intersections, quotients by bad actions,...), as opposed to generic situations (transversal intersections, quotients by free and proper actions,...). (Toën 2014, p. 1)

He explains the need for 'homotopical perturbation' in Kuhnian terms,

...the expression homotopical mathematics reflects a shift of paradigm in which the relation of equality relation is weakened to that of homotopy. (Toën 2014, p. 3)

At the same time he points the reader to the HoTT/UF program as the new foundational language for this homotopical mathematics.

Now, despite this shift to what appears to be the more complex derived setting, familiar features are retained:

Just as an ordinary scheme is defined to be "something which looks locally like $\operatorname{Spec} A$ where $A$ is a commutative ring", a derived scheme can be described as "something which looks locally like $S p e c A$ where $A$ is a simplicial commutative ring." (Lurie 2009, p. 5)

So, an apparently complicated space is being stuck together from pieces. This theme is taken up by Carchedi in a recent paper:

...we will make precise what is means to glue structured $\infty$-topoi along local homeomorphisms (i.e. étale maps) starting from a collection of local models. This parallels the way one builds manifolds out of Euclidean spaces, or schemes out of affine schemes. Since we are allowing our "spaces" to be $\infty$-topoi however, in these two instances we get much richer theories than just the theory of smooth manifolds, or the theory of schemes, but rather get a theory of higher generalized orbifolds and a theory of higher Deligne-Mumford stacks respectively. This same framework extends to the setting of derived and spectral geometry as well. (Carchedi 2013, p. 43)

The obvious point to be made is that all of this is just simply unthinkable without category theory. No category theory, no modern geometry of this kind. On the other hand, it may strike the reader as rather daunting that we may need to get a good handle on what Lurie, Toën and Carchedi are doing with 
$(\infty, 1)$-toposes. However, we are in luck since just the right kind of foundational language is at hand to help. As Toën noted, in recent years there has emerged homotopy type theory (see Shulman and Awodey this volume), which is expected to play the role of the internal language of $(\infty, 1)$-toposes. Now, this language can be extended to describe large tracts of the constructions of geometry. Schreiber found the ingredients for such an extension in the writings of Lawvere, but needed to transplant them from the original topos setting to the setting of $(\infty, 1)$-toposes. Schreiber and Shulman (2014) worked out how this can be done synthetically by adding 'modalities' to homotopy type theory.

I say it is fortunate for us that this is so, but we should not underestimate the work that is still required. If we recall Friedman's scheme of meta-scientific work leading up to a revolution followed by philosophical interpretative work to make sense of it, we might say that the cycle was largely broken through the twentieth century. Even the lessons of the seventy year old category theory are still very far from having been absorbed within philosophy. There have been many contributions made over the decades, but not the kind of sustained work that would make it matter of course for someone entering on a career in philosophy of mathematics to know the basics of category theory. At the very least adjunctions and monads are needed to make any headway.

There will not be space to go into much detail here, but let us begin at the ordinary 1-category level with Lawvere's notion of cohesion (Lawvere 2007) expressed as a chain of adjunctions between a category of spaces and the category of sets. If we take the former to be topological spaces, then one basic mapping takes such a space and gives its underlying set of points. All the cohesive 'glue' has been removed. Now there are two ways to generate a space from a set: one is to form the space with the discrete topology, where no point sticks to another; the other is to form the space with the codiscrete topology, where the points are all glued together into a single blob so that no part is separable, in the sense that there are only constant maps from a codiscrete space to the discrete space with two points. Finally, we need a second map from spaces to sets, one which 'reinforces' the glue by reducing each connected part to an element of a set, the connected components functor, $\pi_{0}$ :

$$
\left(\pi_{0} \dashv \text { Disc } \dashv \dashv \text { coDisc }\right): \text { Top } \rightarrow \text { Set }
$$

These four functors form an adjoint chain, where any of the three compositions of two adjacent functors ( $U \circ c o D i s c, U \circ D i s c, \pi_{0} \circ D i s c$ ) from the category of sets to itself is the identity, whereas, in the other direction, composing adjacent functors to produce endofunctors on Top (coDisc $\circ$ U, Disc $\left.\circ U, D i s c \circ \pi_{0}\right)$ yields two idempotent monads and one idempotent comonad.

Adjoint modalities where the monad is the right adjoint, $\square \dashv \bigcirc$, can be thought of as two different opposite "pure moments", such as codiscreteness and discreteness in this case, or in another example by Lawvere (2000), oddness and evenness of integers. There is an equivalence between types which are pure according to one of the moments with those pure according to the other, but these pure collections inject into the whole differently, as with odd and even 
integers into all integers, and discrete and codiscrete spaces into all spaces.

On the other hand, in adjoint modalities where the monad is on the left, $\bigcirc \square$, there is a single moment, but the full collection of types projects onto those pure according to this moment in two different ways. Here, cohesive spaces project in two opposite ways to discrete spaces, either by the complete removal of the cohesion or by the identication of any cohering points. Another simple example has the real numbers project to the integers (entities which are purely integral) in two ways, via the floor and ceiling functions.

What Schreiber does is to find analogous modalities generated by an adjoint quadruple between an $(\infty, 1)$-topos, $\mathbf{H}$, and the base $(\infty, 1)$-topos of $\infty$ groupoids, $\infty$ Grpd:

$$
(\Pi \dashv \text { Disc } \dashv \Gamma \dashv c o \text { Disc }): \mathbf{H} \rightarrow \infty \text { Grpd } .
$$

The three induced 'adjoint modalities' are called: shape modality $\dashv$ flat modality $\dashv$ sharp modality and denoted $\int \dashv b \dashv \sharp$. In a sense, this $\mathbf{H}$ can be seen as spaces modelled on a 'thickened' point.

Now a very similar pattern repeats itself in the form of a further string of four adjunctions this time between $\mathbf{H}$ and another $(\infty, 1)$-topos, corresponding to extending the thickened point infinitesimally. The three resulting adjoint modalities now comprise two comonads and one monad.

The existence of these two related sets of three adjoint modalities is extraordinarily powerful, allowing the expression of a rich internal higher geometry, including Galois theory, Lie theory, differential cohomology, and Chern-Weil theory, and allows for the synthetic development of higher gauge theory (Schreiber 2013). There remains plenty more interpretative work to be done in making these ideas more accessible, but for our purposes here let us just retain the monad of the second adjoint modality triple, denoted $\Im$ or sometimes $\int_{\text {inf }}$. It is the important one for us to continue the story from Lurie and Carchedi.

So now, despite the apparently intimidating complexity of modern geometry, it is possible to maintain as Schreiber does that there remains a simplicity.

It would seem to me that the old intuition, seemingly falling out of use as the theory becomes more sophisticated, re-emerges strengthened within higher topos theory...Notably all those "generalized schemes," "étale infinity-groupoids" and so forth are nothing but the implementation of the old intuition of "big spaces glued from small model spaces" implemented in homotopy theory...I think it's a general pattern, in the wake of homotopy type theory we find that much of what looks super-sophisticated in modern mathematics is pretty close to the naive idea, but implemented internally in an $\infty$-topos. (Schreiber 2014b)

With homotopy type theory and the six modalities briefly mentioned above, and in particular the infinitesimal shape modality, it is possible to describe synthetically what it is to be a 'formally étale morphism'. Now choosing types, 
$\left\{U_{i}\right\}$, as "model spaces", then a general geometric space is a type $X$ equipped with a map of the form

$$
\coprod_{j} U_{j} \longrightarrow X
$$

such that this map is a 1-epimorphism and formally étale. ${ }^{1}$ We have arrived thus at a synthetic formulation of one of the very basic ideas of geometry.

Of course, there are many such basic ideas for us to consider. In this section I have sketched some ideas of an extraordinarily ambitious body of scientific and meta-scientific work. It may appear that by proposing that we understand cutting-edge geometry, I risk being caught up with the changeable fashions of research, but let us not forget that these projects are rooted in the ideas of Grothendieck from many decades ago, and that later developments were foreseen to some considerable extent by him (see, e.g., Grothendieck 1983). Current ideas thus emerge out of a vast body of work. Indeed, Toën motivates a section where he constructs "a brief, and thus incomplete, history of the mathematical ideas that have led to the modern developments of derived algebraic geometry" as follows:

As we will see the subject has been influenced by ideas from various origins, such as intersection theory in algebraic geometry, deformation theory, abstract homotopy theory, moduli and stacks theory, stable homotopy theory, and so on. Derived algebraic geometry incorporates all these origins, and therefore possesses different facets and can be comprehended from different angles. We think that knowledge of some of the key ideas that we describe below can help to understand the subject from a philosophical as well as from technical point of view. (Toën 2014, p. 6)

If some details will inevitably change, that $(\infty, 1)$-categories lie at the heart of modern geometry will very likely not.

\section{Conclusion}

I have sketched a broad canvas in this chapter. This is to some degree forced upon us by the state we are in where philosophy has drifted from its task. Had the course of philosophy after the famous Davos meeting (Friedman 2000) favoured Cassirer, we might have had a generation of philosophers keen to search for the emergence of new self-understandings in mathematics. Surely in that case category theory, and its higher forms, would have been absorbed much more fully into philosophical consciousness. With the emergence of homotopy type theory, which is already generating considerable philosophical interest, we may see this happen at last. What I have described in this chapter should

\footnotetext{
${ }^{1}$ In the case of schemes, one needs to modify slightly to pro-étale morphisms, in some sense a reflection of the less homogeneous nature of the spaces.
} 
suggest that there is a great deal of further work to be done in coming to understand extensions of homotopy type theory, certainly the cohesive variety so far as geometry goes. It should also be noted that with a linear logic variant of homotopy type theory it is possible to express synthetically many aspects of the quantization of higher gauge theory (Schreiber 2014a).

We have seen Weyl-like meta-scientific work in the formulation of cohesive homotopy type theory, requiring a range of modalities to be added to the basic type theory. Unlike Weyl with Fichte, Schreiber follows Lawvere (1970, 1991) in finding inspiration in Hegel. One can even tell a 'Hegelian' story starting from the opposition between $\emptyset$ and 1, rising through a process of 'Aufhebung' to the six modalities (Schreiber 2014a, Sec 2.4), and even beyond to a further set of three modalities which may be interpreted as capturing the supergeometry needed for dealing with fermions.

Contrast this with a different kind of use of Hegel by Cassirer and also Lakatos, a philosopher more familiar to the Anglophone community. With the new framework for geometry in place, we should be able to tell the Cassirerian story of the unfolding of the past in mathematics and physics, as mathematicians such as Toën are inclined to do by themselves. Mathematics is to be understood by the fact that it constitutes a single tradition of intellectual enquiry. Ideas found at particular stages possess the seeds of later formulations, which retrospectively allow us to understand them better.

We can use this opportunity to gain a grip on some real mathematical content, offering the opportunity for a more interesting dialogue between philosophy of physics and philosophy of mathematics. For one thing, the duality between geometry and algebra that we saw between rings and affine schemes, and which lies behind the relation between the Heisenberg and Schrödinger pictures, continues to higher geometry and higher algebra, where it manifests itself in different formulations of higher gauge theory. Fundamentally, this duality relates to the operation of taking opposities of $(\infty, 1)$-categories.

Finally, as with Cassirer's observation about the seeds of the Erlanger Program lying within our perception, it is sometimes revealed during and after moments of synthesis in mathematics that there is a reliance on aspects of cognition, perception and language, which had possibly gone unnoticed. I think at the very least a form of dependent type theory is present in our cognition as manifested in ordinary language (Ranta 1995). Likewise, the idea of big spaces glued from small model spaces seems very basic. It is surely no accident that mathematicians speak of an "atlas" to define a manifold, since an ordinary atlas provides a collection of maps which overlap. It seems likely we employ something like this in the cognitive maps by which we navigate our domain. Perhaps one of the invariants of geometry has been found here.

\section{Bibliography}

Atiyah, M., 2003. 'What is Geometry?' in The Changing Shape of Geometry: Celebrating a Century of Geometry and Geometry Teaching, Cambridge 
University Press, pp. 24-30.

Ben-Zvi, D., 2014. Blog comment. https://www.math.columbia.edu/ woit/wordpress/?p=7114cpage=1 214353

Borger, J., 2009. Blog comment. https://golem.ph.utexas.edu/category/2009/02/lakatos ${ }_{a} s_{d}$ ialectical $_{r} e$

Carchedi, D., 2013. 'Higher Orbifolds and Deligne-Mumford Stacks as Struc-

tured Infinity Topoi', Arxiv preprint: http://arxiv.org/abs/1312.2204

Cassirer, E., 1944. 'The concept of Group and The Theory of Perception, Philosophy and Phenomenological Research 5(1), pp. 1-36.

Cassirer, E., 1957. The Philosophy of Symbolic Forms: The Phenomenology of Knowledge, Vol. 3, Yale University Press.

Corfield, D., 2003. Towards a Philosophy of Real Mathematics. Cambridge University Press.

Corfield, D., 2012. 'Narrative and the Rationality of Mathematical Practice' in A. Doxiadis \& B. Mazur (eds.) Circles Disturbed: The Interplay of Mathematics and Narrative. Princeton University Press. pp. 244-280.

Corfield, D., 2015. 'Duality as a Category-Theoretic Concept', Studies in History and Philosophy of Modern Physics, doi:10.1016/j.shpsb.2015.07.004.

Domski, M. and Dickson, M. (eds.), 2010. Discourse on a New Method: Reinvigorating the Marriage of History and Philosophy of Science, Open Court Publishing Company.

Einstein, A., 1934. 'On The Method of Theoretical Physics', Philosophy of Science 1(2), pp. 163-169.

Friedman, M., 2000. The Parting of the Ways: Carnap, Cassirer, and Heidegger, Open Court Publishing Company.

Friedman, M., 2001. The Dynamics of Reason, The University of Chicago Press.

Giovanelli, M., 2013. 'Talking at Cross-Purposes: How Einstein and The Logical Empiricists Never Agreed on What They Were Disagreeing About', Synthese 190(17), pp. 3819-3863.

Grothendieck, A., 1983. 'Pursuing stacks', Letter to D. Quillen in G. Maltsiniotis, M. Künzer, and B. Toen (eds.), Documents Mathématiques, Society Mathematics Paris, France

Grothendieck, A. and Verdier, J., 1972. Théorie des topos et cohomologie étale des schémas, Tome 1: Théorie des topos. Lecture Notes in Mathematics, Vol.269. Springer-Verlag, Berlin, SGA 4, M. Artin, A. Grothendieck, and J. L.Verdier (eds.)

Heis, J., 2011. 'Ernst Cassirer's Neo-Kantian Philosophy of Geometry', British Journal for the History of Philosophy 19(4): 759-794.

Lawvere, W., 1970. 'Quantifiers and sheaves', Actes du congres international des mathematiciens, Nice: Vol. 1, pp. 329-334.

Lawvere, W., 1991. 'Some thoughts on the future of category theory', in A. Carboni et. al. (eds.), Category Theory, Springer, pp. 1-13.

Lawvere, W., 2000. 'Adjoint cylinders', Categories mailing list comment, http://permalink.gmane.org/gmane.science.mathematics.categories/1683

Lawvere, W., 2007. 'Axiomatic cohesion', Theory and Applications of Categories 19(3), 41-49. 
Lurie, J., 2009. 'Derived Algebraic Geometry V: Structured Spaces', Arxiv preprint: http://arxiv.org/abs/0905.0459

Marquis, J.-P., 2008. From a Geometric Point of View: a study of the history and philosophy of category theory, Springer.

McLarty, C., 2008. "There is No Ontology Here': Visual and Structural Geometry in Arithmetic', in P. Mancosu (ed.) The Philosophy of Mathematical Practice, Oxford University Press, pp. 370-406.

MRSI, 2014. 'Geometric Representation Theory' Programme announcement, https://www.msri.org/programs/276

Nagel, E., 1939. 'The formation of modern conceptions of formal logic in the development of geometry', Osiris 7, pp. 142-223.

Ranta, A., 1995. Type-Theoretical Grammar, Oxford University Press.

Ryckman, T., 2005. The Reign of Relativity, Oxford University Press.

Scholz, E., 2005. 'Philosophy as a Cultural Resource and Medium of Reflection for Hermann Weyl', Revue de Synthèse 126, pp. 331-351.

Scholz, E., 2011. 'H. Weyl's and E. Cartan's proposals for infinitesimal geometry in the early 1920s', Boletim da Sociedada Portuguesa de Matematica Numero Especial A, pp. 225-245.

Schreiber, U., 2013. 'Differential cohomology in a cohesive infinity-topos', Arxiv preprint: http://arxiv.org/abs/1310.7930

Schreiber, U., 2014a. 'Quantization via Linear homotopy types', Arxiv preprint: http://arxiv.org/abs/1402.7041.

Schreiber, U., 2014b, nForum discussion comment, http://nforum.ncatlab.org/discussion/2084/highergeometry $/$ ?Focus $=49931$ Comment 49931

Schreiber, U. and Shulman, M., 2014. 'Quantum gauge field theory in cohesive homotopy type theory', Arxiv preprint: http://arxiv.org/abs/1408.0054

Toën, B., 2014. 'Derived algebraic geometry', Arxiv preprint: http://arxiv.org/abs/1401.1044.

Torretti, R., 1978. Philosophy of Geometry from Riemann to Poincaré, Springer. 